\title{
Myristic Acid
}

National Cancer Institute

\section{Source}

National Cancer Institute. Myristic Acid. NCI Thesaurus. Code C68393.

A saturated long-chain fatty acid with a 14-carbon backbone. Myristic acid is found naturally in palm oil, coconut oil and butter fat. 